\title{
Direct and Indirect Gradient Control for Static
}

\section{Optimisation}

\author{
Yi Cao \\ School of Engineering, Cranfield University, U.K.
}

Keywords: Plantwide Control, Self-Optimising Control, Control Schemes, Decentralised Control, Cascade Control, Automatic Differentiation.

\begin{abstract}
Static "self-optimising" control is an important concept, which provides a link between static optimisation and control (Skogestad, 2000). According to the concept, a dynamic control system could be configured in such a way that when a set of certain variables are maintained at their setpoints, the overall process operation is automatically optimal or near optimal at steady-state in the presence of disturbances. A novel approach using constrained gradient control to achieve "self-optimisation" has been proposed by Cao (2004). However, for most process plants, the information required to get the gradient measure may not be available in real-time. In such cases, controlled variable selection has to be carried out based on measurable candidates. In this work, the idea of direct gradient control has been extended to controlled variable selection based on gradient sensitivity analysis (indirect gradient control). New criteria, which indicate the sensitivity of the gradient function to disturbances and implementation errors, have been derived for selection. The
\end{abstract}


particular case study shows that the controlled variables selected by gradient sensitivity measures are able to achieve near optimal performance.

\section{Introduction}

Chemical process plants are always controlled in different layers. For example, several local control layers are designed to maintain local controlled variables at the desired operating point whilst a plantwide optimisation layer is responsible to adjust the setpoint to the local layers according to different situations (disturbances). Traditionally, these two layers are designed separately for different (economic and dynamic) objectives although they need working together. However, these two layers can be linked together via the concept of "self-optimising control", which can date back to the work of Morari et al. (1980) about "feedback optimising control", and has been revisited recently by Skogestad (2000). Self-optimisation is a control strategy where by controlling certain specially selected variables at their nominal setpoints, the overall system automatically achieves the optimal (or acceptable) operating conditions without re-optimisation even in the presence of disturbances.

The optimality of a self-optimising control system is strongly related to the control structure, particularly the controlled variables selected. For controlled variable selection, Morari et al. (1980) proposed a second-order derivative criterion, whilst Skogestad (2000) derived a criterion using the minimum singular value index. Both works are based on a common assumption that the first-order gradient of the cost function is zero at optimal point. However, this assumption is questionable. Firstly, the gradient of cost function may not equal to zero at a constrained local optimum. Secondly, without directly control, the gradient will vary away from zero in the presence of disturbances even if it is zero under a nominal condition. In spite of this deficiency, the concept of "self-optimising" control has successfully 
been applied to several chemical processes such as the Tennessee Eastman process (Larsson et al., 2001) and the evaporation process of Newell and Lee (Govatsmark and Skogestad, 2001).

To overcome the above deficiency, Cao (2004) has derived a dimension reduced expression for the gradient of a constrained cost function, and used it as the controlled variable to achieve self-optimising control. In this work, the usefulness of the dimension-reduced gradient function is scrutinised further. It is shown that the sensitivities of the gradient function to disturbances and to implementation errors for a set of controlled variables (maintained at constant) are effective and reliable criteria for controlled variable selection. By applying these measures to the evaporation process of Newell and Lee, a new controlled variable is identified to be the best and simplest one for self-optimising control. The effectiveness of this new controlled variable is demonstrated through simulation.

The paper is organised in a self-contained way: The reduced-dimension gradient of the constrained cost function as a combination of the first-order derivatives of the cost function and nonlinear model functions is represented in section 2. Then the sensitivities of the dimension-reduced gradient to disturbances and to implementation errors for a set of controlled variables are derived in section 3 . A general structure of "self-optimising" control is discussed in section 4, where a special cascade control structure is proposed to cope with conditionally active constraints such that both optimality and constraint conditions are satisfied in the same system. The sensitivity measures as criteria for controlled variable selection are applied to the evaporation process in section 5, where several controlled variables are identified as the best and simplest solutions. A comparison based on static and dynamic simulation performed for different controlled variable configurations is presented. Finally, the paper is concluded in section 6. 


\section{Active constraints and dimension-reduced gradient}

Consider the following optimisation problem:

$$
\begin{array}{cc}
\min _{x, u} J & =\phi(x, u, d) \\
\text { s.t. } & f(x, u, d)=0 \\
& g(x, u, d) \leq 0
\end{array}
$$

where $x \in \mathbb{R}^{n_{x}}, u \in \mathbb{R}^{n_{u}}$ and $d \in \mathbb{R}^{n_{d}}$ are state, input and disturbance variables respectively. For a given disturbance, $d$, the solution of the above optimisation problem is denoted as, $x^{*}$ and $u^{*}$. Assume that at the optimal point, the following equalities hold:

$$
F\left(x^{*}, u^{*}, d\right)=\left[\begin{array}{c}
f\left(x^{*}, u^{*}, d\right) \\
g_{1}\left(x^{*}, u^{*}, d\right)
\end{array}\right]=0
$$

where $f(\cdot)$ and $g_{1}(\cdot)$ are vector-valued functions with dimensions of $n_{f}$ and $n_{1}$ respectively. If $m=\left(n_{x}+n_{u}\right)-\left(n_{f}+n_{1}\right) \neq 0$, then according to the Kuhn-Tucker conditions, there are $m$ first-order optimal conditions. Denote $u^{=}\left[\begin{array}{ll}u_{1}^{T} & u_{2}^{T}\end{array}\right]^{T}$ with $u_{2} \in \mathbb{R}^{m}, z=\left[\begin{array}{ll}x^{T} & u_{1}^{T}\end{array}\right]^{T}$ and $v=u_{2}$. Then the optimisation problem $[1]$ can be restated as:

$$
\begin{aligned}
\min _{z, v} J= & \phi(z, v, d) \\
\text { s.t. } & F(z, v, d)=0
\end{aligned}
$$

The first-order optimal conditions of the above optimisation problem are:

$$
\begin{aligned}
J_{v}=\phi_{v}+\frac{\partial z}{\partial v} \phi_{z} & =0 \\
F_{v}+\frac{\partial z}{\partial v} F_{z} & =0
\end{aligned}
$$


If the Jacobian matrix, $F_{z}$ is not singular, then the second condition (5) gives:

$$
\frac{\partial z}{\partial v}=-F_{v} F_{z}^{-1}
$$

Inserting (6) into the first condition (4) leads to the following m-dimension optimal condition:

$$
G(z, v, d):=\phi_{v}-F_{v} F_{z}^{-1} \phi_{z}=0
$$

Normally, the left-hand-side of the above condition is a function of $x, u\left(u_{1}\right.$, and $\left.u_{2}\right)$ and $d$. For a given disturbance, $d$, equation (7) corresponds to an unique solution of $v^{*}=u_{2}^{*}$, from which all rest system variables, $x^{*}$ and $u_{1}^{*}$ can be determined.

If $F\left(x^{*}, u^{*}, d\right)=0$ is the only active constraints for all possible disturbances, then it is clear that $G(z, v, d)=0$ is the only condition which must be maintained to ensure the process operation is optimal. In other words, if condition $G(z, v, d)=0$ is retained by the control system (direct gradient control), then optimal operation can be achieved without re-optimisation for different disturbances, i.e. the plant is self-optimising controlled.

\section{Gradient sensitivity measures}

Direct gradient control requires $G(z, v, d)$ available online. However, the gradient normally is a function of the system's states, inputs and disturbances. Some of these variables may not be measured in a real plant. Hence, direct gradient control cannot be implemented in such a system. In this case, a set of measured variables as the substitute of the gradient has to be selected for "self-optimising" control. 


\subsection{Sensitivity to disturbances}

When other variables rather than the gradient itself are retained by a control system, the gradient in (7) is a function of disturbances and will not always be zero. The magnitude of the gradient indicates the optimality of the operation. Therefore, it is desirable to select controlled variables, which make the gradient as insensitive to disturbances as possible. The sensitivity of the gradient to disturbances depends on which $m$ controlled variables selected. Assume $m$ controlled variables selected correspond to $m$ equations denoted as, $H(z, v, d)=0$, then the sensitivity can be derived from the following equation set:

$$
\begin{aligned}
\delta & =G(z, v, d) \\
0 & =F(z, v, d) \\
0 & =H(z, v, d)
\end{aligned}
$$

Sensitivities of (9) and (10) to disturbances are zero, i.e.

$$
\begin{aligned}
\frac{\partial z}{\partial d} F_{z}+\frac{\partial v}{\partial d} F_{v}+F_{d} & =0 \\
\frac{\partial z}{\partial d} H_{z}+\frac{\partial v}{\partial d} H_{v}+H_{d} & =0
\end{aligned}
$$

Since $F_{z}$ is not singular, equation (11) leads to

$$
\frac{\partial z}{\partial d}=-\left(\frac{\partial v}{\partial d} F_{v}+F_{d}\right) F_{z}^{-1}
$$

Inserting (13) into the second equation $(12)$ leads to:

$$
\frac{\partial v}{\partial d}=-\left(H_{d}-F_{d} F_{z}^{-1} H_{z}\right)\left(H_{v}-F_{v} F_{z}^{-1} H_{z}\right)^{-1}
$$


Replacing $\frac{\partial v}{\partial d}$ in 13 with 14 gives:

$$
\begin{aligned}
\frac{\partial z}{\partial d}= & -F_{d} F_{z}^{-1}+ \\
& \left(H_{d}-F_{d} F_{z}^{-1} H_{z}\right)\left(H_{v}-F_{v} F_{z}^{-1} H_{z}\right)^{-1} F_{v} F_{z}^{-1}
\end{aligned}
$$

Using the results in $(14)$ and $(15)$, the sensitivity of $\delta$ to disturbances can be yielded as follows:

$$
\begin{aligned}
\delta_{d}= & \frac{\partial z}{\partial d} G_{z}+\frac{\partial v}{\partial d} G_{v}+G_{d} \\
= & \left(G_{d}-F_{d} F_{z}^{-1} G_{z}\right)-\left(H_{d}-F_{d} F_{z}^{-1} H_{z}\right) \\
& \left(H_{v}-F_{v} F_{z}^{-1} H_{z}\right)^{-1}\left(G_{v}-F_{v} F_{z}^{-1} G_{z}\right)
\end{aligned}
$$

where $\delta_{d} \in \mathbb{R}^{n_{d} \times m}$. When $H=G, \delta_{d}=0$. This corresponds to direct gradient control. For other controlled variables, $H \neq G$, normally $\delta_{d} \neq 0$. The $i$-th row norm of $\delta_{d}$ matrix indicates how sensitive of the gradient to the $i$-th disturbance for the $m$ controlled variables selected. Therefore, the row norm of $\delta_{d}$ can be used as a selection criterion to rank different controlled variable combinations.

The sensitivity measure, $\delta_{d}$ is a second-order derivative, $J_{v d}$ of the constrained cost function. At the nominally optimal point, as explained in (Skogestad, 2000), the first-order derivative of cost function is zero. Second-order derivatives must be used to compare different controlled variable combinations. However, the minimum singular value measure, proposed as a selection criterion in (Skogestad, 2000) is only part of a second-order derivative. Therefore, it can only give a biased prediction. In contradiction to the minimum singular value measure, the sensitivity measure introduced here is a complete second-order derivative and can provide unbiased comparison for alternatives. Another important feature of the sensitivity function, $\delta_{d}$ is that it is independent of the scaling of controlled variables. Therefore, a comparison based on $\delta_{d}$ is more objective than that based on the minimum 
singular value measure, which is scaling dependent.

\subsection{Sensitivity to implementation error}

Similar sensitivity analysis can also be applied to evaluate the gradient sensitivity to measurement noise, to model uncertainties and to implementation errors. As an example, the gradient to implementation error is considered in this section.

Denote implementation errors, $\varepsilon \in \mathbb{R}^{m}$, which associate with $m$ controlled equations as:

$$
0=\tilde{H}(z, v, d, \varepsilon)=H(z, v, d)-\varepsilon
$$

It leads to $\tilde{H}_{z}=H_{z}, \tilde{H}_{v}=H_{v}, \tilde{H}_{d}=H_{d}$ and $\tilde{H}_{\varepsilon}=-I$. In equation (16) replace $d$ with $\varepsilon$ and $H$ with $\tilde{H}$ respectively and consider $F_{\varepsilon}=0$ and $G_{\varepsilon}=0$ (process equilibrium, active constraints and theoretic gradient are independent of $\varepsilon$ ). Then, the gradient sensitivity with respect to the implementation errors is derived as follows:

$$
\delta_{\varepsilon}=\left(H_{v}-F_{v} F_{z}^{-1} H_{z}\right)^{-1}\left(G_{v}-F_{v} F_{z}^{-1} G_{z}\right)
$$

where $\delta_{\varepsilon} \in \mathbb{R}^{m \times m}$. Particularly, when, $H=G, \delta_{\varepsilon}=I$, i.e. $\delta=\varepsilon$.

\subsection{Sensitivity calculation}

For a small system, the gradient function, $G(z, v, d)$ can be derived analytically. Therefore, the sensitive measures, $\delta_{d}$ and $\delta_{\varepsilon}$ can be calculated by linearisation of the plant model. Assume the nonlinear model equations and the gradient function 
are linearised around the nominally optimal point as follows:

$$
\begin{aligned}
\dot{x} & =A x+B_{1} u_{1}+B_{2} u_{2}+B_{3} d \\
y_{1} & =C_{1} x+D_{11} u_{1}+D_{12} u_{2}+D_{13} d \\
y_{2} & =C_{2} x+D_{21} u_{1}+D_{22} u_{2}+D_{23} d \\
\delta & =C_{3} x+D_{31} u_{1}+D_{32} u_{2}+D_{33} d
\end{aligned}
$$

where $y_{1}$ corresponds to active constraints of $g_{1}(x, u, d)=0$ and $y_{2}$ is controlled variables selected for self-optimising control. Then the Jacobian matrices required to calculate $\delta_{d}$ can be obtained from the above system matrices by using the following equalities:

$$
\begin{aligned}
& F_{z}=\left[\begin{array}{ll}
A & B_{1} \\
C_{1} & D_{11}
\end{array}\right]^{T} \quad F_{v}=\left[\begin{array}{c}
B_{2} \\
D_{12}
\end{array}\right]^{T} \quad F_{d}=\left[\begin{array}{c}
B_{3} \\
D_{13}
\end{array}\right]^{T} \\
& H_{z}=\left[\begin{array}{ll}
C_{2} & D_{21}
\end{array}\right]^{T} \quad H_{v}=D_{22}^{T} \quad H_{d}=D_{23}^{T} \\
& G_{z}=\left[\begin{array}{ll}
C_{3} & D_{31}
\end{array}\right]^{T} \quad G_{v}=D_{32}^{T} \quad G_{d}=D_{33}^{T}
\end{aligned}
$$

Particularly, for systems without active constraints, i.e. $n_{1}=0$, matrices $B_{1}, C_{1}$, $D_{11}, D_{12}, D_{13}$ and $D_{21}$ are empty. Denote steady-state gain matrices between different signals at the nominally optimal point as, $L_{y v}=D_{22}-C_{2} A^{-1} B_{2}, L_{y d}=$ $D_{23}-C_{2} A^{-1} B_{3}, L_{G v}=D_{32}-C_{3} A^{-1} B_{2}$ and $L_{G d}=D_{33}-C_{2} A^{-1} B_{3}$. Then the sensitivity measures can be simplified as:

$$
\begin{aligned}
\delta_{\varepsilon}^{T} & =L_{G v} L_{y v}^{-1} \\
\delta_{d}^{T} & =L_{G d}-L_{G v} L_{y v}^{-1} L_{y d}
\end{aligned}
$$

The above equations clearly show how sensitivity measures are associated with the minimum singular value measure, $\underline{\sigma}\left(L_{y v}\right)=1 /\left\|L_{y v}^{-1}\right\|$. If the system has no active 
constraints, or all active constraints have been implicitly included in equilibrium equations, and manipulated variables have been properly scaled such that $L_{G v}=I$, then $\delta_{\varepsilon}$ is equivalent to the minimum singular value measure. Further more, only when $L_{G d}=0$ (no explicit dependence of gradient on disturbances) and disturbances are also properly scaled such that $L_{y d}=I$, then equivalency between $\delta_{d}$ and the minimal singular value measure is true. Otherwise, if $L_{G d} \neq 0$, the minimal singular value measure can only partially predict self-optimising properties.

For a large or complicated process, it may not be possible to get analytical expression of the gradient function. In that case, the sensitivity measures, $\delta_{d}$ and $\delta_{\varepsilon}$ can still be numerically calculated as the second-order derivatives, $J_{v d}$ and $J_{v \varepsilon}$ of the constrained cost function. For this purpose, the recently developed automatic differentiation techniques (Griewank, 2000) can play an important role.

\section{Conditionally active constraints}

Controlled variables in a self-optimising plant should include: stabilising variables related to plant unstable modes, active constraint variables included in $g_{1}=0$ in (2), self-optimising variables, $G$ or those with small $\delta_{d}$ and $\delta_{\varepsilon}$. However, active constraints of a process plant may not always be the same. Some output constraints, such as temperature and pressure limits may becomes active under certain circumstances. Traditionally, for safety reasons, these variables are always selected as controlled variables. However, by controlling these variables at their nominal setpoints, the plant operation will not be optimal at most times.

To satisfy both requirements of self-optimisation and operating constraints, a cascade control structure is proposed as shown in Figure 1. In Figure 1, an inner loop is closed for constraint control. The setpoint of the inner loop is determined via a saturation block by the outer loop, which is designated for self-optimising 
control by maintaining the self-optimising variable at constant. Within the feasible range of the process constraint, the setpoint of the inner loop is floating as a manipulated variable to perform self-optimising control. However, when disturbances cause the process towards outside of the constraints, the saturation block will limit the setpoint within the constraint so that the controlled variable of the inner loop will be kept within feasible range. In this way, the self-optimising control and constraint control loops alternatively become active and inactive to achieve constrained self-optimisation.

\section{Evaporator case study}

\subsection{Gradient function}

The new controlled variable selection approach is applied to an evaporation process (Newell and Lee, 1989), shown in Figure 2

This is a "forced-circulation" evaporator, where the concentration of dilute liquor is increased by evaporating solvent from the feed stream through a vertical heat exchanger with circulated liquor. The process variables are listed in Table 1 and model equations are given in Appendix A.

The economic objective is to minimise the operational cost $[\$ / \mathrm{h}]$ related to steam, cooling water and pump work (Heath et al., 2000; Wang and Cameron, 1994):

$$
J=600 F_{100}+0.6 F_{200}+1.009\left(F_{2}+F_{3}\right)
$$

The process has the following constraints related to product specification, operation 
safety and design limits:

$$
\begin{aligned}
X_{2} & \geq 35+0.5 \% \\
40 \mathrm{kPa} \leq \quad P_{2} & \leq 80 \mathrm{kPa} \\
P_{100} & \leq 400 \mathrm{kPa} \\
F_{200} & \leq 400 \mathrm{~kg} / \mathrm{min} \\
0 \mathrm{~kg} / \mathrm{min} \leq \quad F_{3} & \leq 100 \mathrm{~kg} / \mathrm{min}
\end{aligned}
$$

Note a $0.5 \%$ back-off has been enforced on $X_{2}$ to ensure the variable remaining feasible for all possible disturbances. The process model has three state variables, $L_{2}, X_{2}$ and $P_{2}$ with eight degrees of freedom. Four of them are disturbances, $F_{1}$, $X_{1}, T_{1}$ and $T_{200}$. The rest four degrees of freedom are manipulable variables, $F_{2}$, $P_{100}, F_{3}$ and $F_{200}$. The optimisation problem of 25] with process constraints, 26] to 30 has been solved under nominal disturbances:

$$
d=\left(\begin{array}{llll}
F_{1} & X_{1} & T_{1} & T_{200}
\end{array}\right)^{T}=\left(\begin{array}{llll}
10 & 5 & 40 & 25
\end{array}\right)^{T}
$$

The minimum cost obtained is $6178.2 \$ / \mathrm{h}$ and corresponding values of process variables are shown in Table 1 .

At the optimal point, there are two active process constraints, $X_{2}=35.5 \%$ and $P_{100}=400[\mathrm{kPa}]$. These two constraints will keep active within whole disturbance region, which is defined as $\pm 20 \%$ of the nominal disturbances. Physically, the first active constraint is because a higher outlet composition requires more solvent to be evaporated, therefore needs more steam, cooling water and pump cost. For the second constraint, since heater duty, $Q_{100}$ is determined by both steam pressure, $P_{100}$ and circulating flowrate, $F_{3}$, reducing $P_{100}$ will increase $F_{3}$ due to energy balance. However, the sensitivity to steam cost of $P_{100}$ is much lower than that of $F_{3}$. Hence, an optimal operation should keep $X_{2}$ at its lower bound and $P_{100}$ at its higher bound. 
These two active constraints plus the separator level, which has no steady-state effect on the plant operation, but must be stabilised at its nominal setpoint, consume three degrees of freedom. Therefore, the optimal condition has one degree of freedom. Choose cooling water flowrate, $F_{200}$ as $v$ and rest manipulated variables and state variables as $z$, i.e.

$$
z=\left(\begin{array}{llllll}
L_{2} & X_{2} & P_{2} & F_{2} & P_{100} & F_{3}
\end{array}\right)^{T}
$$

By using (7), the following gradient function is obtained:

$$
\begin{aligned}
G= & 0.6-0.5538 \frac{T_{201}-T_{200}}{F_{200}} \times \\
& \left(6.306 \frac{0.16\left(F_{1}+F_{3}\right)+0.07 F_{1}}{T_{100}-T_{2}}+\frac{42 F_{1}}{36.6}\right)
\end{aligned}
$$

\subsection{Self-optimising variable selection}

The nonlinear gradient function, (32) requires both disturbances, $F_{1}$ and $T_{200}$ measured online. If one of them, or both of them are not measured in real-time, then an alternative measurement need to be selected to achieve self-optimisation. It can be selected from the set of all measurable and manipulable variables. The process has twelve measurements and four manipulated variables. Three of them, $L_{2}, X_{2}$ and $P_{100}$ has already been selected for stabilising and constraint control. Amount the rest variables, $F_{2}, F_{4}, F_{5}$ have to be determined by the equilibrium of the system and $T_{2}, T_{3}, P_{100}, Q_{100}$ and $Q_{200}$ are dependent on some other variables. Therefore, only five variables represent independent alternatives: $P_{2}, F_{100}, T_{201}, F_{200}$ and $F_{3}$. The authors of (Govatsmark and Skogestad, 2001) have considered another controlled variable, $F_{200} / F_{1}$. In addition, a new controlled variable, $T_{201}-T_{200}$ is also considered in this work. The gradient sensitivity measures to four disturbances and to implementation errors are calculated (see Table 2) using (16) and (18) with 
disturbances and controlled variables both scaled by $20 \%$ of their nominal values.

Table 2 shows that if neither $F_{1}$ nor $T_{200}$ is measured online, then $T_{201}$ and $F_{200}$ are two most promising choices. $T_{201}$ is better when $F_{1}$ and $X_{1}$ are main disturbances, but $F_{200}$ becomes better when $T_{200}$ is the dominant disturbance. However, when either $F_{1}$ or $T_{200}$ is available in real-time, $F_{200} / F_{1}$ or $T_{201}-T_{200}$ are the best controlled variables with minor difference. Implementation error is the dominant factor affecting optimality for these two choices. It is also expected that choosing either $T_{201}-T_{200}$ or $F_{200} / F_{1}$ will be as good as controlling the gradient.

\subsection{Simulation results and comparison}

Top four most promising controlled variables listed in Table 2 plus the gradient function in (32) are compared with constant $P_{2}$ control by static and dynamic simulation. For static simulation, 1000 sets of disturbances are randomly generated within feasible range. Static responses to these disturbances for the six control schemes are obtained. The mean value of the corresponding costs are calculated and shown in the first column of Table 5

For dynamic simulation, all six control schemes are implemented in a decentralised cascade structure: $L_{2}$ controlled by $F_{3}, X_{2}$ controlled by $F_{2}$, one of the five controlled variables controlled by the setpoint of $P_{2}$, which is in turn controlled by $F_{200}$ to satisfy both the self-optimising and conditionally active constraint control as shown in Figure 1

Three loops are controlled by PI controllers with parameters shown in in Table 3. whilst self-optimising variables in all schemes are controlled with constant static gains: 1000 for $G$ and $F_{200} / F_{1}$ loops, 20 for $T_{201}-T_{200}$ and $T_{201}$ loops, and 10 for $F_{200}$ loop.

In the simulation, all disturbances are modelled as a step signal passing through a first-order delay. The amplitudes of step changes are randomly produced within 
the $\pm 20 \%$ range of the nominal values. The changing intervals and time constants of the first-order delays are different for different disturbance variables shown in Table 4

With the above configuration, simulation for a 20-hour operation is performed. The total operation costs of the six schemes are shown in the second column of Table 5

It is shown in Table 5 that costs of all four most promising schemes are very close to the cost using $G$ in both static and dynamic simulation. This demonstrates the concept of self-optimising control, i.e. optimal or near optimal plant operation can be achieved by selecting certain controlled variables to be controlled at constant setpoints. The relative ranking of alternative controlled variables is almost coincident with the prediction of the sensitivity measure (Table 2) except that in Table 5 the schemes using $F_{200} / F_{1}$ is slightly better than using $T_{201}-T_{200}$. To explain the difference between these two configurations, the dynamic simulation results of three best schemes, using $G$, using $F_{200} / F_{1}$ and using $T_{201}-T_{200}$ are compared in Figure 3 .

From Figure 3 it can been seen that the cascade control structure works well in all three schemes. When pressure constraint of $P_{2}$ is inactive, self-optimising control is active, the gradient response has very small deviation in all three schemes. However, when $P_{2}$ reaches $40[\mathrm{kPa}]$ at 3.5 and 19.5 hour, out control loops become inactive, hence large deviations of self-optimising variables are observed. Particularly, the scheme using $T_{201}-T_{200}$ has larger offset than the one using $F_{200} / F_{1}$ when $P_{2}$ constraint is active. The offset in scheme using $T_{201}-T_{200}$ is also more sensitive to controller gain than the one in scheme using $F_{200} / F_{1}$. Therefore, control gain of the former has to be much smaller than the one of the latter to limit the maximal deviation. However, the smaller the control gain the larger the average deviation, i.e. the larger the implementation error. Therefore, the loos of objective 
function using $T_{201}-T_{200}$ is larger than the one using $F_{200} / F_{1}$ due to different implementation error although the gradient sensitivity to implementation error is the same for both schemes.

\section{Conclusions}

The concept of self-optimising control has been scrutinised. Based on the direct gradient control described in (Cao, 2004), the sensitivities of the gradient function to disturbance and to implementation error have been derived and proposed as criteria for controlled variable selection in self-optimising control system design (indirect gradient control). The sensitivity measure is a second-order derivative of the cost function and is independent of measurement scaling. Therefore, it can provide objective and unbiased comparison for controlled variable selection. The gradient sensitivity can be calculated from the linearised model when the gradient is available analytically, or numerically calculated by applying the newly developed automatic differentiation techniques. The evaporator case study demonstrates the effectiveness of this new selection measure. Two better controlled variables are able to be identified by using these sensitivity criteria. The case study also demonstrates the success of using cascade control to cope with conditionally active constraints.

\section{Acknowledgements}

The work is supported by the EPSRC UK under grant GR/R57324. 


\section{References}

Cao, Y. (2004). Constrained self-optimizing control via differentiation. In: Prepresents of the 7th International Symposium on Advanced Control of Chemical Processes (ADCHEM). Hong Kong. pp. 63-70.

Govatsmark, M.S. and S. Skogestad (2001). Control structure selection for an evaporation process. In: Proceedings of ESCAPE'11. Kolding, Denmark. pp. 657662.

Griewank, A. (2000). Evaluating Derivatives. SIAM. Philadelphia, PA.

Heath, J.A., I.K. Kookos and J.D. Perkins (2000). Process control structure selection based on economics. AICHE J. 46(10), 1998-2016.

Larsson, T., K. Hesterun, E. Hovland and S. Skogestad (2001). Self-optimization control of a large-scale plant: The tennessee eastman process. Ind. Eng. Chem. Res. 40, 4889-4901.

Morari, M., Y. Arkun and G. Stephanopoulos (1980). Studies in the synthesis of control structures for chemical process, Part I: Formulation of the problem. Process decomposition and the classification of the control tasks. Analysis of the optimizing control structures.. AIChE Journal 26(2), 220-232.

Newell, R.B. and P.L. Lee (1989). Applied Process Control - A Case Study. Prentice Hall. Englewood Cliffs, NJ.

Skogestad, S. (2000). Plantwide control: the search for the self-optimizing control structure. J. Process Control 10(5), 487-507.

Wang, F.Y. and I.T. Cameron (1994). Control studies on a model evaporation process - constrainted state driving with conventional and higher relative degree systems. J. Process Control 4(2), 59-75. 


\section{A Model equations}

$$
\begin{aligned}
\frac{\mathrm{d} L_{2}}{\mathrm{~d} t} & =\frac{F_{1}-F_{4}-F_{2}}{20} \\
\frac{\mathrm{d} X_{2}}{\mathrm{~d} t} & =\frac{F_{1} X_{1}-F_{2} X_{2}}{20} \\
\frac{\mathrm{d} P_{2}}{\mathrm{~d} t} & =\frac{F_{4}-F_{5}}{4} \\
T_{2} & =0.5616 P_{2}+0.3126 X_{2}+48.43 \\
T_{3} & =0.507 P_{2}+55.0 \\
F_{4} & =\frac{Q_{100}-0.07 F_{1}\left(T_{2}-T_{1}\right)}{38.5} \\
T_{100} & =0.1538 P_{100}+90.0 \\
Q_{100} & =0.16\left(F_{1}+F_{3}\right)\left(T_{100}-T_{2}\right) \\
F_{100} & =Q_{100 / 36.6} \\
Q_{200} & =\frac{0.9576 F_{200}\left(T_{3}-T_{200}\right)}{0.14 F_{200}+6.84} \\
T_{201} & =T_{200}+\frac{13.68\left(T_{3}-T_{200}\right)}{0.14 F_{200}+6.84} \\
F_{5} & =\frac{Q_{200}}{38.5}
\end{aligned}
$$




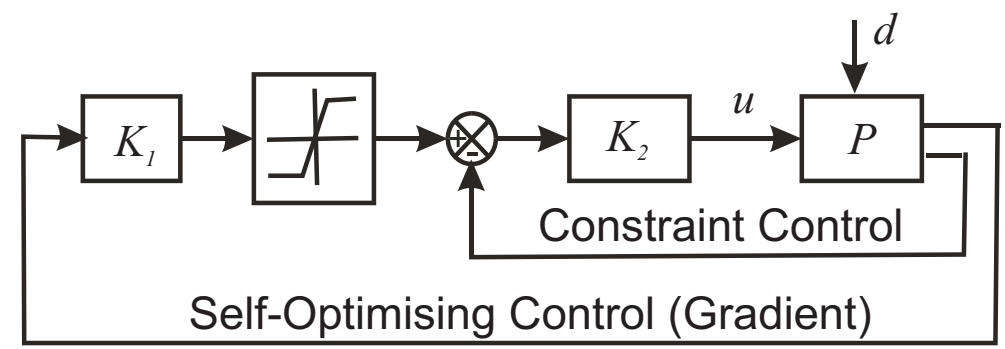

Figure 1: Cascade structure for self-optimising and constraint control 


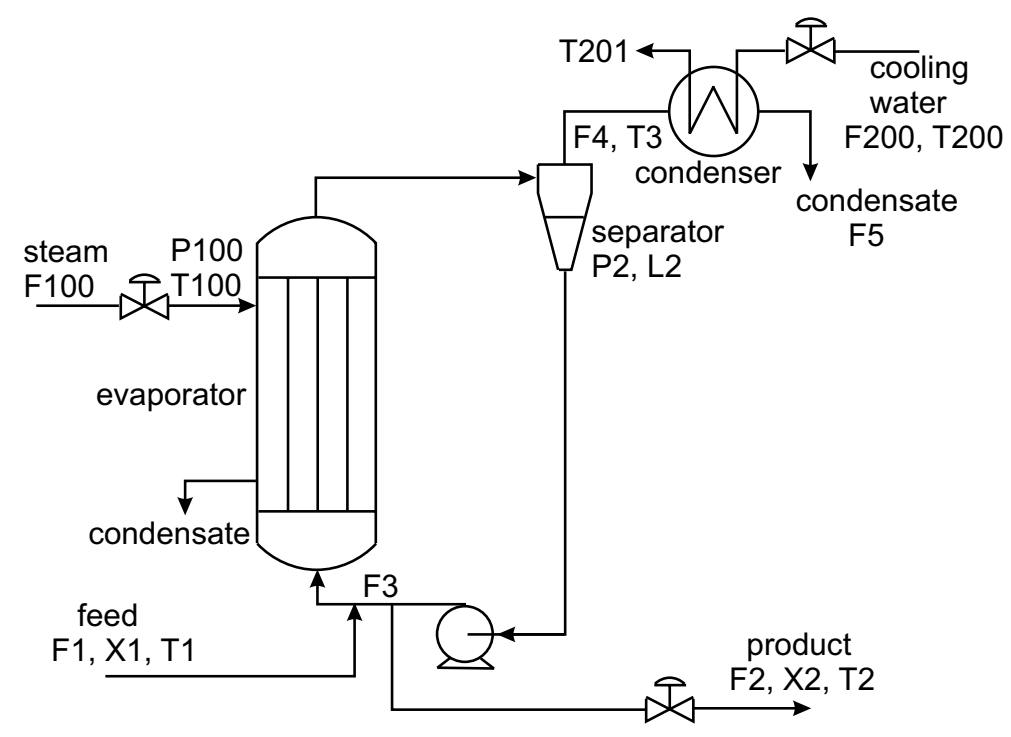

Figure 2: Evaporator System 
(a)

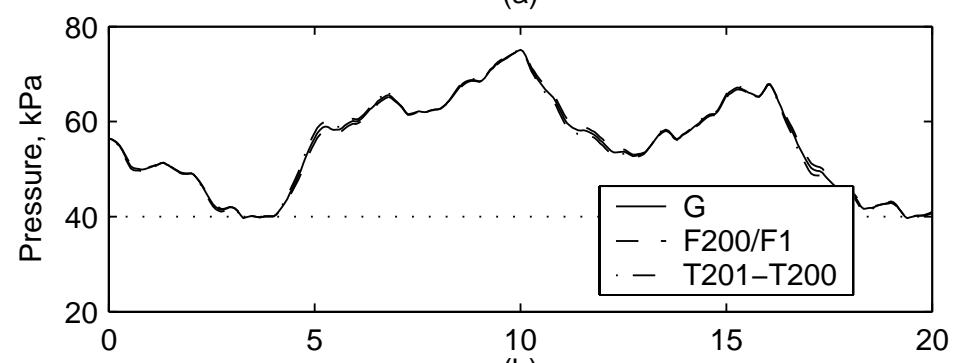

(b)

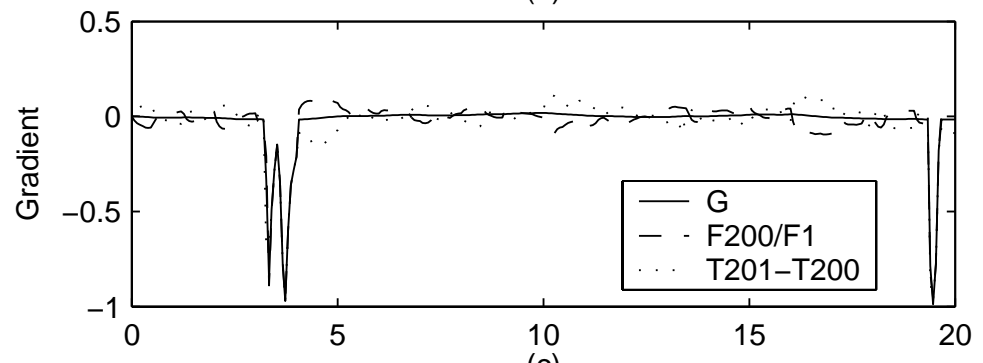

(c)

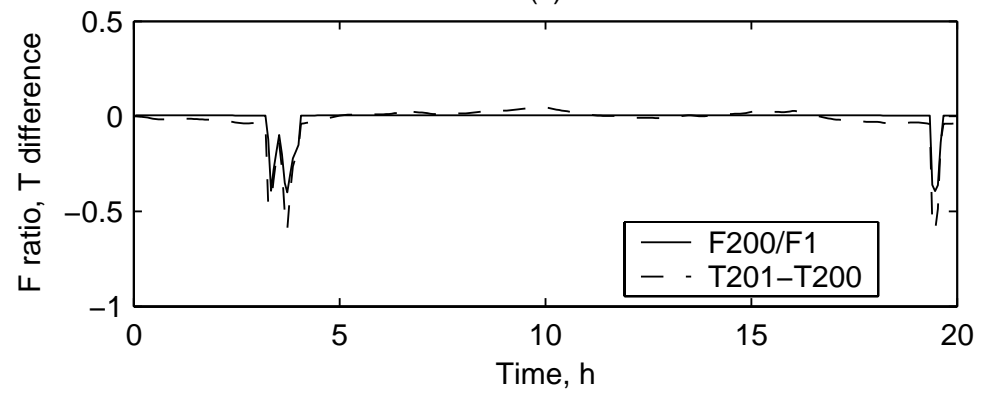

Figure 3: Simulation result comparison. (a) $P_{2}$ response. (b) $G$ response. (c)scaled deviation of $F_{200} / F_{1}$ and $-\left(T_{201}-T_{200}\right)$ from nominal steady-state. 
Table 1: Variables and Optimal Values

\begin{tabular}{llrl}
\hline Variable & Description & Value & Unit \\
\hline$F_{1}$ & Feed flowrate & 10 & $\mathrm{~kg} / \mathrm{min}$ \\
$F_{2}$ & Product flowrate & 1.41 & $\mathrm{~kg} / \mathrm{min}$ \\
$F_{3}$ & Circulating flowrate & 23.05 & $\mathrm{~kg} / \mathrm{min}$ \\
$F_{4}$ & Vapour flowrate & 8.59 & $\mathrm{~kg} / \mathrm{min}$ \\
$F_{5}$ & Condensate flowrate & 8.59 & $\mathrm{~kg} / \mathrm{min}$ \\
$X_{1}$ & Feed composition & $\%$ \\
$X_{2}$ & Product composition & 35.5 & $\%$ \\
$T_{1}$ & Feed temperature & 40 & ${ }^{\circ} \mathrm{C}$ \\
$T_{2}$ & Product temperature & 91.22 & ${ }^{o} \mathrm{C}$ \\
$T_{3}$ & Vapour temperature & 83.61 & ${ }^{\circ} \mathrm{C}$ \\
$L_{2}$ & Separator level & 1 & $\mathrm{~meter}$ \\
$P_{2}$ & Operating pressure & 56.42 & $\mathrm{kPa}$ \\
$F_{100}$ & Steam flowrate & 10.02 & $\mathrm{~kg} / \mathrm{min}$ \\
$T_{100}$ & Steam temperature & 151.52 & ${ }^{\circ} \mathrm{C}$ \\
$P_{100}$ & Steam pressure & 400 & $\mathrm{kPa}$ \\
$Q_{100}$ & Heat duty & 366.63 & $\mathrm{~kW}$ \\
$F_{200}$ & Cooling water flowrate & 230.54 & $\mathrm{~kg} / \mathrm{min}$ \\
$T_{200}$ & Inlet cooling water temperature & 25 & ${ }^{\circ} \mathrm{C}$ \\
$T_{201}$ & Outlet cooling water temperature & 45.5 & ${ }^{\circ} \mathrm{C}$ \\
$Q_{200}$ & Condenser duty & 330.77 & $\mathrm{~kW}$ \\
\hline
\end{tabular}


Table 2: Sensitivity measures of alternative controlled variables against disturbances and implementation errors

\begin{tabular}{cccccc}
\hline C.V. & $\delta_{F_{1}}$ & $\delta_{X_{1}}$ & $\delta_{T_{1}}$ & $\delta_{T_{200}}$ & $\delta_{\varepsilon}$ \\
\hline$T_{201}-T_{200}$ & 0.0124 & 0.0167 & 0.0005 & 0.0064 & 0.2426 \\
$F_{200} / F_{1}$ & 0.0124 & 0.0231 & 0.0005 & 0.0064 & 0.2426 \\
$T_{201}$ & 0.0124 & 0.0167 & 0.0005 & 0.2895 & 0.5385 \\
$F_{200}$ & 0.2550 & 0.0231 & 0.0005 & 0.0064 & 0.2426 \\
$P_{2}$ & 1.1324 & 0.2044 & 0.0005 & 0.5854 & 0.6772 \\
$F_{3}$ & 1.9840 & 0.3753 & 0.0878 & 0.5854 & 0.8516 \\
$F_{100}$ & 12.326 & 1.8600 & 0.8544 & 0.5854 & 11.1936 \\
\hline
\end{tabular}


Table 3: PI controller parameters

\begin{tabular}{ccc}
\hline Loop & Gain & Integral time $[\mathrm{min}]$ \\
\hline$\left(L_{2}, F_{3}\right)$ & 200 & 5 \\
$\left(X_{2}, F_{2}\right)$ & 36.74 & 4.6619 \\
$\left(P_{2}, F_{200}\right)$ & 200 & 6.667 \\
\hline
\end{tabular}


Table 4: Disturbance model parameters

\begin{tabular}{ccc}
\hline Disturbance & Interval [min] & Time constant [min] \\
\hline$F_{1}$ & 120 & 20 \\
$X_{1}$ & 6 & 2 \\
$T_{1}$ & 15 & 5 \\
$T_{200}$ & 15 & 5 \\
\hline
\end{tabular}


Table 5: Alternative controlled variables and operating costs

\begin{tabular}{ccc}
\hline self-optimising c.v. & Static Mean $[\$]$ & 20h Dynamic cost $[\$]$ \\
\hline$G$ & 6139.80 & 120,823 \\
$F_{200} / F_{1}$ & 6139.83 & 120,826 \\
$T_{201}-T_{200}$ & 6139.82 & 120,828 \\
$F_{200}$ & 6141.08 & 120,854 \\
$T_{201}$ & 6142.42 & 120,857 \\
$P_{2}$ & 6162.57 & 121,561 \\
\hline
\end{tabular}

